\title{
Spatial knowledge acquisition from maps and from navigation in real and virtual environments
}

\author{
ANTHONY E. RICHARDSON, DANIEL R. MONTELLO, and MARY HEGARTY \\ University of Califormia, Santa Barbara, California
}

\begin{abstract}
In this study, the nature of the spatial representations of an environment acquired from maps, navigation, and virtual environments (VEs) was assessed. Participants first learned the layout of a simple desktop VE and then were tested in that environment. Then, participants learned two floors of a complex building in one of three learning conditions: from a map, from direct experience, or by traversing through a virtual rendition of the building. VE learners showed the poorest learning of the complex environment overall, and the results suggest that $\mathrm{VE}$ learners are particularly susceptible to disorientation after rotation. However, all the conditions showed similar levels of performance in learning the layout of landmarks on a single floor. Consistent with previous research, an alignment effect was present for map learners, suggesting that they had formed an orientation-specific representation of the environment. VE learners also showed a preferred orientation, as defined by their initial orientation when learning the environment. Learning the initial simple VE was highly predictive of learning a real environment, suggesting that similar cognitive mechanisms are involved in the two learning situations.
\end{abstract}

People generally acquire environmental spatial knowledge through direct experience by locomoting through an environment or by viewing a map. A third way is to view a simulation of the environment, displayed in a slide or a video presentation or, most recently, in a virtual environment (VE). Considerable experimental evidence shows that map learning and direct environmental learning produce qualitatively different spatial representations (e.g., Evans \& Pezdek, 1980; Thorndyke \& Hayes-Roth, 1982). One of the goals of this study is to further explore this phenomenon by carefully controlling participant exposure to the environment and by using multiple measurement techniques to produce converging lines of evidence. The use of VEs for spatial knowledge acquisition is relatively new and still poorly understood. Specifically, it is unknown whether people also construct qualitatively different representations from this learning mode. A second goal of this study is to examine the spatial representations formed from VE learning and to determine whether knowledge acquired in a VE transfers to a real-world setting. A final goal is to examine the relationship between learning in real environments and that in VEs - specifically, whether the same abilities are required in these different learning experiences.

\section{Spatial Representations Acquired From Different Learning Experiences}

Navigation and maps. A classic study by Thorndyke and Hayes-Roth (1982) compared spatial learning from

This work was supported by Award DASW01-95-K-0014 from the Army Research Institute for Behavioral Science and Social Sciences. The authors are grateful to Lance Rushing for development of the virtual environment and to Michael Provenza for assisting in data collection. Correspondence concerning this article should be addressed to A. E. Richardson, Department of Geography, University of California, Santa Barbara, CA 93106 (e-mail: tonyr@geog.ucsb.edu). maps with that from direct navigation. Participants learned the layout of a building, either by studying a map or by working and navigating in the building. Map learners estimated route distances and straight-line distances equally well, whereas navigation learners estimated route distances more accurately than straight-line distances. Map learners were less accurate in pointing to unseen locations in the building but were more accurate in placing locations on a map, relative to locations in the building. Thorndyke and Hayes-Roth concluded that, after studying a map, people have a survey, or bird's-eye, representation of the environment, from which they can directly estimate straight-line distances. However, they make more errors in pointing to unseen locations in the building, because of the difficulty of translating the overhead perspective of the map to a horizontal one. In contrast, they proposed that, after navigating in a building, people have procedural knowledge of routes between locations in the building. This provides an explicit representation of route distance, but straight-line distance must be computed from this route distance, which adds error to the estimate.

A possible weakness of Thorndyke and Hayes-Roth's (1982) study is that the participants in their map and navigation conditions had very different amounts of exposure to the environment learned: Their exposure varied from minutes to an hour in the map condition and from 1 month to 2 years in the navigation condition. Furthermore, the landmarks in the building had more significance for the navigation learners, since these landmarks were part of their everyday work environment. In the present study, we controlled prior familiarity and learning time, in order to compare learning from a map with learning from navigation, given equal amounts of exposure to each environment.

Virtual environments. Learning from a VE is similar to learning from navigation, since the interface preserves 
many of the visual-spatial characteristics experienced during real navigation and the learner takes a horizontal perspective in the environment, building up a spatial representation over time through movement within the environment. Therefore, it is interesting to ask whether people form the same types of spatial representations from navigating in VEs and in real environments and whether learning the layout of an environment from a VE transfers to navigation performance in the real environment.

To date, research on the effectiveness of VEs in training has had mixed results. Witmer, Bailey, Knerr, and Parsons (1996) found that VE training was useful in a building navigation task but did not produce the same level of performance as map or real-world training. Bliss, Tidwell, and Guest (1997) found no difference between map and VE training in a similar task but did not compare performance to real-environment training, and Philbin, Ribarsky, Walker, and Hubbard (1998) found map training to be superior to VE training. Other studies have shown evidence for learning and successfully navigating solely within a VE (Darken \& Sibert, 1996; Regian, Shebilske, \& Monk, 1992). Ruddle, Payne, and Jones (1997) trained and tested participants in a virtual rendition of the original study environment used by Thorndyke and HayesRoth (1982). After 10 learning trials, participants showed levels of orientation and navigation performance similar to those of the original building navigators.

Transfer of training from a VE to a real environment probably depends on the extent of similarity between the virtual and real learning situations. This similarity is dependent on the type of VE in question. Computer-simulated environments can vary along several dimensions, such as whether interaction in the environment is learner controlled or passive, whether presentation of information in the display is multimodal (e.g., visual, auditory, proprioceptive) or unimodal, and the degree of immersion in the environment (Wickens \& Baker, 1995). In this study, interaction with the environment was learner controlled with a keyboard interface, but followed a specific route through the environment, as directed by the experimenter. Information was displayed visually on a computer monitor. The degree of immersion in the environment was low, relative to systems using head-mounted stereoscopic displays.

Although desktop VEs are more similar to real worlds than are maps or video simulations, they nevertheless differ in important respects. One important difference is the lack of proprioceptive cues during navigation. Navigation in a desktop VE produces an optic/vestibular mismatch of information; optic flow is available from the display specifying forward or lateral motion (Warren \& Hannon, 1988), but vestibular senses indicate a stationary position. Another difference is that, in a desktop VE, the user must make a scale translation from the small world displayed on the screen to the real world. Preliminary evidence suggests that distance and the size of an environment is often underestimated in VEs (Henry \& Furness, 1993; Witmer $\&$ Kline, 1998). Finally, the field of view in a desktop VE eliminates most peripheral vision, which may be nec- essary for the development of survey level knowledge (Alfano \& Michel, 1990; Sholl, 1996).

\section{Alignment Effects in Spatial Representations}

One of the most striking differences between spatial representations constructed from different learning experiences is the presence of alignment effects. When an individual learns an environment from a map and is then tested in the environment, he or she is typically very accurate when aligned with the original orientation of the map and shows poor performance when contra-aligned (Levine, Marchon, \& Hanley, 1984). This effect is not produced after learning an environment directly (Evans \& Pezdek, 1980). These findings suggest that map learning produces orientation-specific representations of the environment.

Presson and Hazelrigg (1984) suggest that the presence of alignment effects depends on whether environments are learned by directly viewing the environment (primary learning) or from a symbolic medium, such as a map (secondary learning). More recent studies have suggested that differences in alignment effects reflect the number of orientations from which the environment is viewed during learning (Lloyd \& Cammack, 1996; MacEachren, 1992). When people are exposed to maps from multiple orientations, as compared with a single orientation, alignment effects are eliminated. However, their representations are less precise, as is evidenced by greater pointing errors or longer reaction times. Furthermore, Shelton and McNamara (1997) reported that when people learned the layout of a real environment from two different viewpoints (primary learning), they appeared to construct two orientation-specific representations. Alignment effects can also occur when learning an environment directly under conditions of limited sensory information (Palij, Levine, \& Kahan, 1984; Presson, DeLange, \& Hazelrigg, 1987, 1989), such as when participants are blindfolded or maintain a constant orientation in the environment, so that they do not receive rotational vestibular feedback (because they do not turn while walking in the environment).

It is interesting to examine the existence of alignment effects in a VE, since a VE is a type of secondary learning in which an environment is viewed from multiple perspectives and no vestibular information is available. In the present study, we tested for alignment effects with respect to the initial direction faced in the environment when learning the real environment or the VE.

\section{Predicting Individual Differences in Environmental Spatial Learning}

A final goal of the present study was to examine whether the ability to learn the layout of a VE is predictive of the ability to learn the layout of a different environment by navigation or from a map. To date, there have been only a few attempts to assess individual differences in larger scale or environmental spatial ability (Bryant, 1982; Goldin \& Thorndyke, 1981, 1982; Lorenz \& Neisser, 
1986; Pearson \& Ialongo, 1986). Furthermore, these studies found only weak correlations between pencil-andpaper tests of spatial abilities and measures of environmental spatial ability, such as learning the layout of a novel environment. This led Lorenz and Neisser to conclude that "conventional psychometric measures of mental spatial manipulation are clearly distinct from naturalistic measures of geographic direction awareness, landmark memory, and route knowledge" (p. 28). There is currently no psychometric spatial abilities test that is a good predictor of environmental spatial ability. In the present experiment, all the participants learned a simple VE by navigation, and we examined how individual differences in this learning task predicted individual differences in learning a more complex environment from a map or from navigation in a real environment or a VE. If learning a simple VE is predictive of individual differences in largescale environmental learning tasks, it might afford a useful way of examining environmental spatial abilities in the laboratory.

\section{METHOD}

\section{Participants}

Sixty-one undergraduate students at the University of California, Santa Barbara, participated in the study. The participants were enrolled in an introductory geography course and received extra credit for their participation. They were between 18 and 49 years of age (mean, 22.5 years). Equal numbers of males and females were assigned to each condition.

\section{Materials}

Direction estimates were made with a rigid circular dial with a wire pointer. The dial measured $25 \mathrm{~cm}$ in diameter. A single radius line, visible on top of the dial, was used to orient the dial to the participant, and direction estimates were read off of the bottom of the dial, which was marked in single degree increments.

Simple virtual environment. A simple VE was constructed, using the Doom II game engine, created by Id Software. It was displayed by a Pentium-120 on an SVGA 15 -in. monitor. The simple environment consisted of four corridors and three turns, with no doors or windows. Three landmarks were included: a barrel in the first corridor, a candelabra in the third corridor, and an exit sign at the end of the fourth corridor. A map of the simple VE is shown in Figure 1. Movement in the environment was initiated with the four arrow keys, enabling the user to look left and right or to move forward and back.

Complex environment. Ellison Hall, a building on the campus of the University of California, Santa Barbara, was chosen as the learning environment. As is shown in Figures $2 \mathrm{~A}$ and 2B, Ellison Hall is a six-floor $\mathrm{H}$-shaped building. The participants learned a route covering approximately $300 \mathrm{ft}$ through the second and third floors. There were eight designated landmarks along the route, four on each floor. The landmarks on the second floor were (1) the Asian American Studies sign, (2) a bookcase, (3) a red door alarm sign, and (4) a chair. The landmarks on the third floor were (5) the geography department sign, (6) a wall-sized world map, (7) a gray door, and (8) the political science mailroom sign. Landmarks were chosen for their distinctiveness in the environment. For example, the chair was a large upholstered chair unlike any in the building, and the gray door was located in a hallway in which all the other doors were green.

Virtual Rendition. As with the simple environment, the simulated building was created as a Doom II environment. The building

\section{Landmark 3}

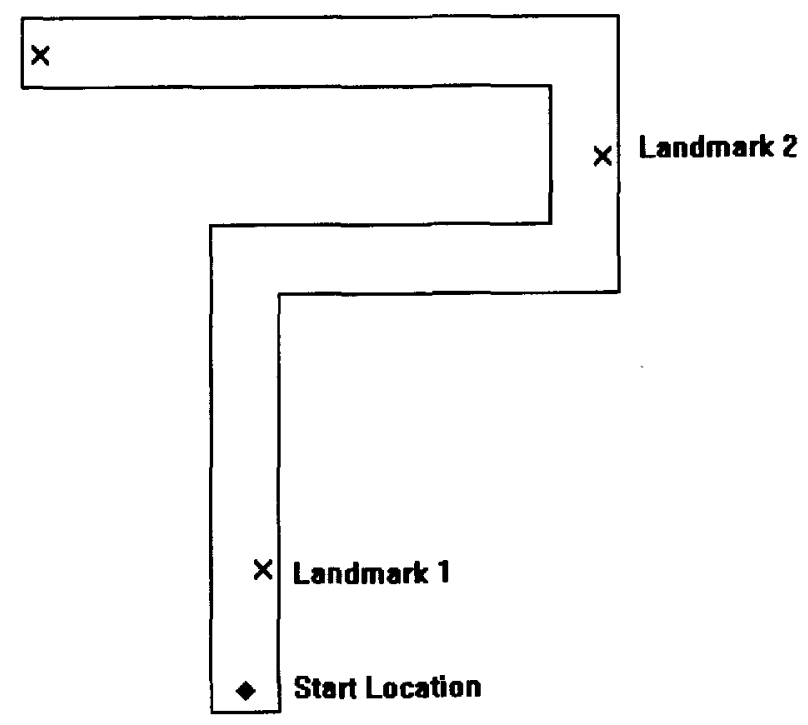

Figure 1. Layout of the simple virtual environment.

was constructed to scale and contained objects found in the real environment; however, it was quite impoverished in comparison with the real building. Eight landmarks were created that corresponded to landmarks in the real building.

Map. Maps of the second and third floors of the building were created by scanning the building blueprints. All detail was eliminated, except for the stairwells and the eight landmarks. Each landmark was labeled with an arrow pointing to its exact location. These maps are shown in Figures 2A and 2B.

Questionnaire. A questionnaire was administered, to determine factors relevant to performance on the tasks. It comprised six 7-point Likert-scale questions. Two questions assessed the participants' knowledge of the experimental setting and of the campus in general. Three questions referred to their experience while traveling in the VE, and one question assessed their experience playing 3-D virtual reality type games.

\section{Procedure}

There were four phases of the experiment: (1) simple VE learning and testing, (2) complex environment learning, (3) complex environment testing, and (4) the final questionnaire. In Phase 2, the participants acquired knowledge about the layout of the building via one of three methods: by walking through the real building, by moving through a virtual rendition of the building, or by examining a map. The participants were randomly assigned to a learning condition and were tested individually.

Simple virtual environment learning. In the first phase, the participants in all the conditions learned the layout of a simple VE. The participants first spent time familiarizing themselves with the keyboard and practicing moving about in a practice VE in the shape of a closed loop. When the participants declared that they were comfortable with the interface, practice was terminated. Next, the participants learned the layout of the simple VE. They were instructed to pay close attention to how far they walked and to the location of the landmarks that they encountered. To give them a sense of scale, they were informed that the practice loop that they had walked was $160 \mathrm{ft}$ for an entire circuit. The participants were instructed to move along the corridor, and the experimenter pointed out and named the three landmarks along the way. They traversed the route twice. On the third pass, they were asked to pause facing each landmark and to indicate the route distance, straight-line distance, and direction to 
A

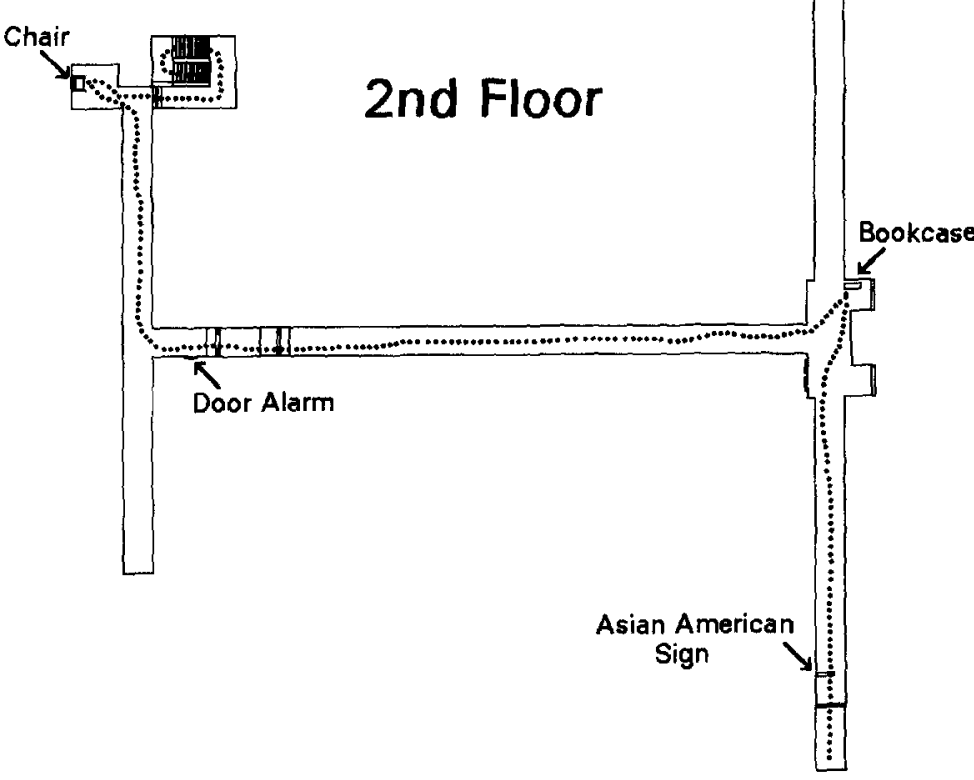

B
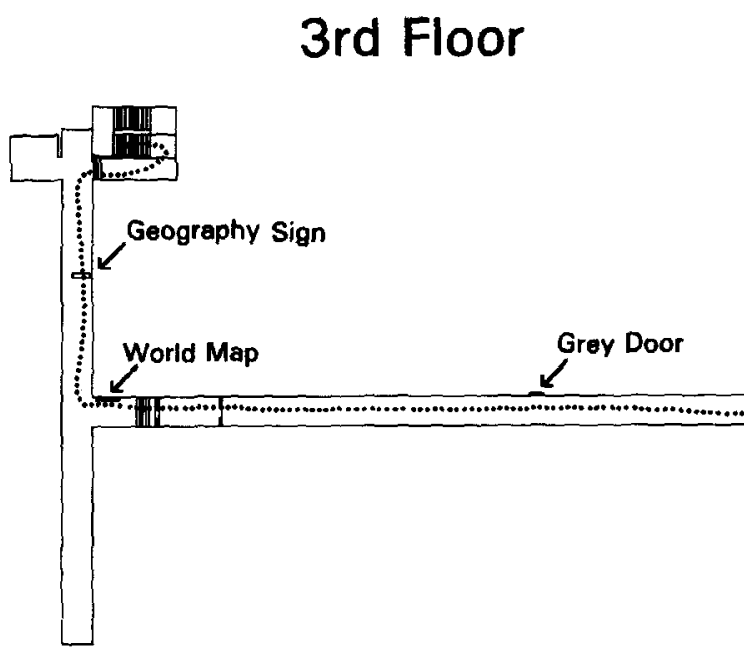

Political Science Sign

Figure 2. (A) Layout of the second floor of the complex environment (dotted line indicates path traveled by the participants and was not seen by the map learners). (B) Layout of the third floor of the complex environment.

the other two landmarks. Distance estimates were made in feet; directions were indicated with the rigid circular dial.

Complex environment learning. The second phase consisted of learning the building layout by walking through the actual building, moving in the VE, or examining the map. The route required 6 min to traverse in both the real environment and the VE; total map learning exposure was also $6 \mathrm{~min}$. In all the conditions, half of the participants began the route at Landmark 1 (the Asian American
Studies sign $)$ and learned the route and landmarks in the order listed above. The other half began at Landmark 8 (the political science mailroom sign) and learned the route and landmarks in the reverse order. The participants were informed that the purpose of the study was to determine how much spatial knowledge people can acquire in an environment and that their task was to learn the layout of the route and to remember the names and locations of each of the eight landmarks. 
In the real-navigation condition, the participants were led to the starting point--the second floor balcony on the southwest corner of the building or the third floor balcony on the southeast corner. They were instructed to follow the experimenter and to pay attention to the landmarks encountered. At each landmark, the experimenter paused to indicate the name, and the participant was asked to repeat the names of the previous landmarks in their correct order. Errors were noted and corrected by the experimenter. At the end of the route, the experimenter began testing (Phase 3 ).

In the VE condition, the participants began at the same (virtual) balcony in the building. The speed of movement through the VE was slowed so as to be equal to normal walking speed, in order that total building exposure would be equal to that of the real environment. Verbal instructions were used to direct the participant throughout the building. As in the previous condition, at each landmark, the participants were requested to name the previous landmarks.

In the map condition, the participants learned the layout by viewing the map of the building projected on a $2-\mathrm{m}$ screen by an overhead projector. The route was drawn on a transparency of the map, and each landmark was specified as it was encountered. In addition to naming the landmark, the experimenter also indicated with an arrow the direction the participant would be facing at each landmark. Half of the arrows pointed in alignment with the map (to the top) and half were misaligned by $90^{\circ}$ or $180^{\circ}$. At each landmark, the map transparency was removed from the overhead projector, and the participant was requested to name the previous landmarks. When the experimenter completed the route on the first floor, the participants were told that they would be climbing (or descending) a stairway to the next floor and that they should study the layout for the next $30 \mathrm{sec}$. Next, the map transparency of the subsequent floor was returned to the overhead projector, and the route along that floor was delineated in the same manner.

Complex environment testing. In the first part of the testing phase, the participants were required to make spatial judgments while imagining that they were within the environment. The participants were positioned at a table and instructed to imagine that they were directly facing each of the landmarks and then made route distance, straight-line distance, and direction estimates to other landmarks. The participants were instructed to ignore the vertical dimension of the building and to point to landmarks as if they were all on the same level. Route distance estimates included distance along the entire path, including stairways. To provide a sense of scale, the participants were told that the width of the hallway was $9 \mathrm{ft}$. All the distance estimates were made in feet.

In the second phase, the participants were led back into the real building for in situ testing. The participants in all the conditions were tested in the real building in this phase. The experimenter led them to each landmark and again asked for distance and direction judg. ments to other landmarks. For the map and VE participants, this was their first exposure to the real environment. The participants made 28 route distance, 28 straight-line distance, and 28 pointing judgments, equally divided between the imagination and the in situ testing phases.

In the last phase of the experiment, the participants were administered the questionnaire.

\section{RESULTS}

\section{Complex Environment Directional Estimates}

Directional estimates were analyzed by using an analysis of variance (ANOVA) on absolute pointing error and by assessing the separate contributions of constant and variable error to this absolute error (Schutz \& Roy, 1973). Constant error was not large in this study, and vari- able error results mirrored those of absolute error, so only absolute error results will be presented and discussed. No differences in pointing error were found between those who started on the second floor and those who started on the third floor $[F(1,58)=0.09]$, so the data for these two conditions were combined for subsequent analysis. All post hoc comparisons reported are significant at the .05 level, using Tukey's studentized range test.

Overall, the participants in the VE condition had a significantly higher absolute error $\left(M=57.8^{\circ}, S D=50.9^{\circ}\right)$ than those in the map $\left(M=41.4^{\circ}, S D=49.4^{\circ}\right)$ and the realwalk $\left(M=41.6^{\circ}, S D=47.0^{\circ}\right)$ conditions $[F(2,58)=$ $3.70, p<.05]$. Post hoc comparisons indicated no significant overall difference between the map and the real conditions. However, significant interactions with other factors provide a more complete account of the differences between conditions.

As is shown in Figure 3, there was a significant interaction between learning condition and whether or not the participants were pointing to landmarks within the same floor or between the two floors $[F(2,58)=6.49, p<.01]$. Post hoc comparisons indicated that, for within-floor pointing, the differences in errors were not statistically significant across the three learning conditions. However, the VE condition had significantly higher errors when pointing between floors than did both the map and the real-walk conditions.

A marginal interaction was found between learning condition and whether responses were made while imagining being in the environment or whether they were made in situ $[F(2,58)=2.83, p<.10]$. As is shown in Figure 4, all the conditions had a greater error in the imagination

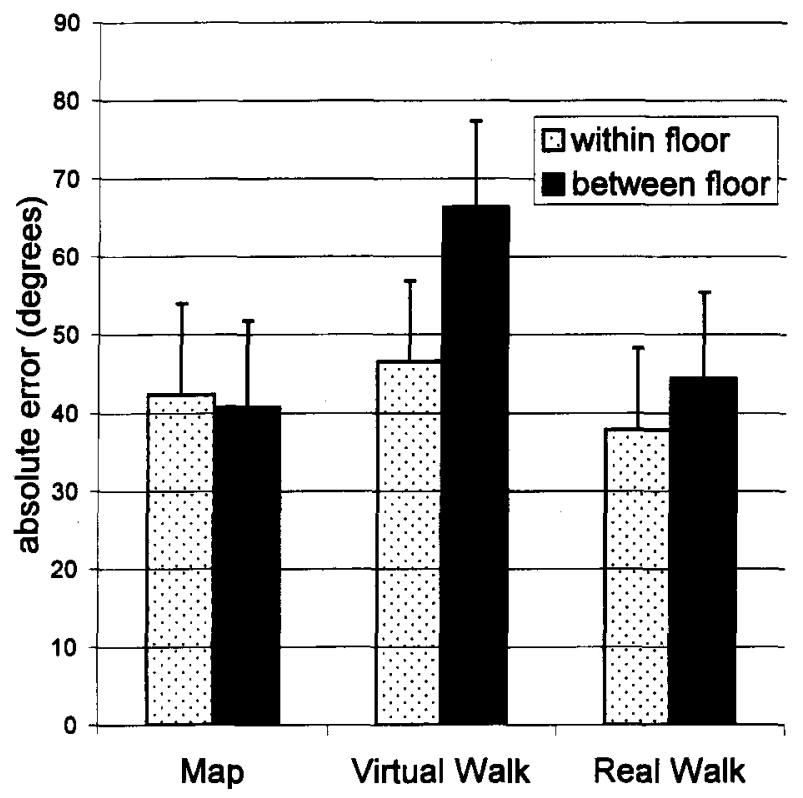

Figure 3. Absolute pointing error for within-floor versus betweenfloor responses (error bars represent one standard error). 


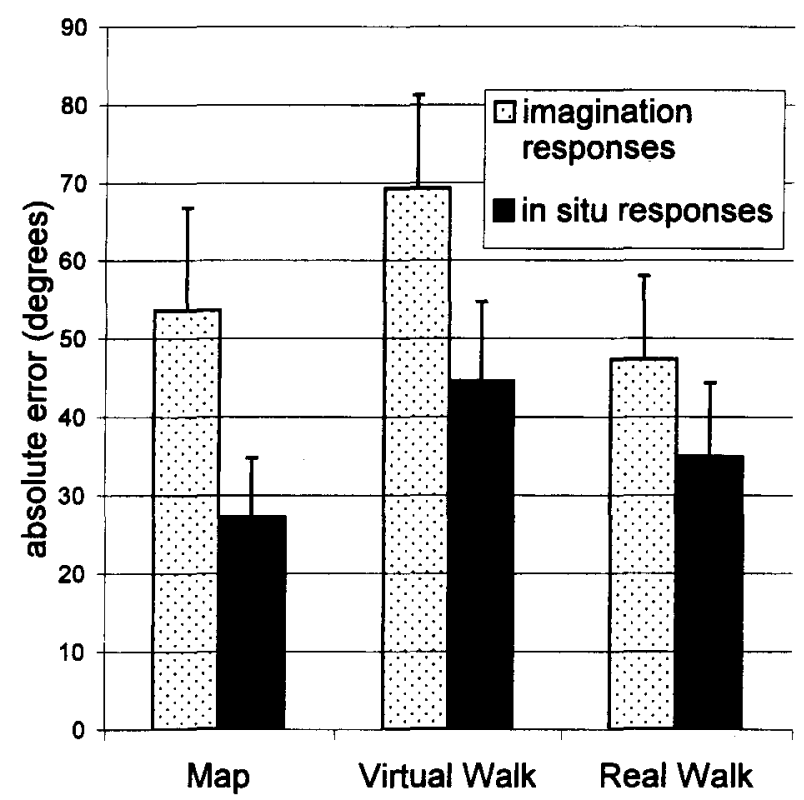

Figure 4. Absolute pointing error for imagined versus in situ responses (error bars represent one standard error).

condition than in the in situ condition; however, this difference was greater for the participants in the map and VE conditions than for the participants in the real-walk condition.

Alignment effects. Next, evidence for a map alignment effect is examined. The participants at each testing location either imagined that they were facing the landmark with a specific orientation (in the imagination condition) or were positioned to face the landmark with that same orientation (in the in situ condition). If that orientation corresponded to the orientation of the map, the orientation was classified as aligned; otherwise, it was classified as misaligned. On the basis of previous research (e.g., Evans \& Pezdek, 1980), we predicted poorer performance in the misaligned conditions for the map group. However, alignment defined in this way is not meaningful for the VE and real-walk learners, and so no alignment effect is predicted for these conditions. As Figure 5 shows, a significant interaction was found between learning condition and map alignment $[F(2,58)=8.92, p<.001]$. Post hoc comparisons indicate that, as was predicted, there was a significant difference in pointing error between aligned and misaligned orientations in the map condition and no such difference in the VE and real-walk conditions.

Although there is no reason to expect a map alignment effect for participants who learned from the real walk or the VE, it is possible that the participants in these conditions formed a representation that was aligned with the initial starting orientation of the route- -that is, the direction faced when beginning the first leg of the real or the virtual walk. Therefore, we conducted another analysis on just these two learning conditions, in which orientation was classified as aligned if it corresponded to the starting position orientation for the virtual- and real-walk conditions. As is shown in Figure 6, there was a marginally significant interaction between learning condition and starting orientation alignment $[F(1,58)=2.88, p<.10]$. Post hoc comparisons show a significant difference between aligned and misaligned orientations for the VE condition and no significant difference for the real-walk condition. Since the participants started at two different locations, there were two starting position orientations, offset by $180^{\circ}$. An alignment effect was found for both of these starting orientations.

\section{Complex Environment Distance Estimates}

Individuals are likely to have different conceptions of standard distances; these data provide supporting evidence. Although the average estimated route distance from start to finish was $457 \mathrm{ft}$, estimates ranged as high as 3,000 ft. Given the variance in absolute estimates of distance, a more suitable way of examining such data is through a relative distance measure (Montello, 1991). Therefore, the correlation between objective distance and estimated distance was used to assess distance estimation performance. Correlations were first transformed to $z$ scores, using Fisher's $r$-to- $z$ transformation, and these $z$ scores were contrasted in a repeated measures ANOVA.

A main effect was found for estimation type. As Figure 7 shows, route distance correlations were higher than straight-line distance correlations $[F(2,58)=21.47, p<$ $.001]$. In addition, there was a learning condition $\times$ estimation type interaction $[F(2,58)=4.32 p<.05]$. Post hoc comparisons indicated that map learners were equally accurate in their route and straight-line distance estimates, whereas VE and real-walk learners were more accurate

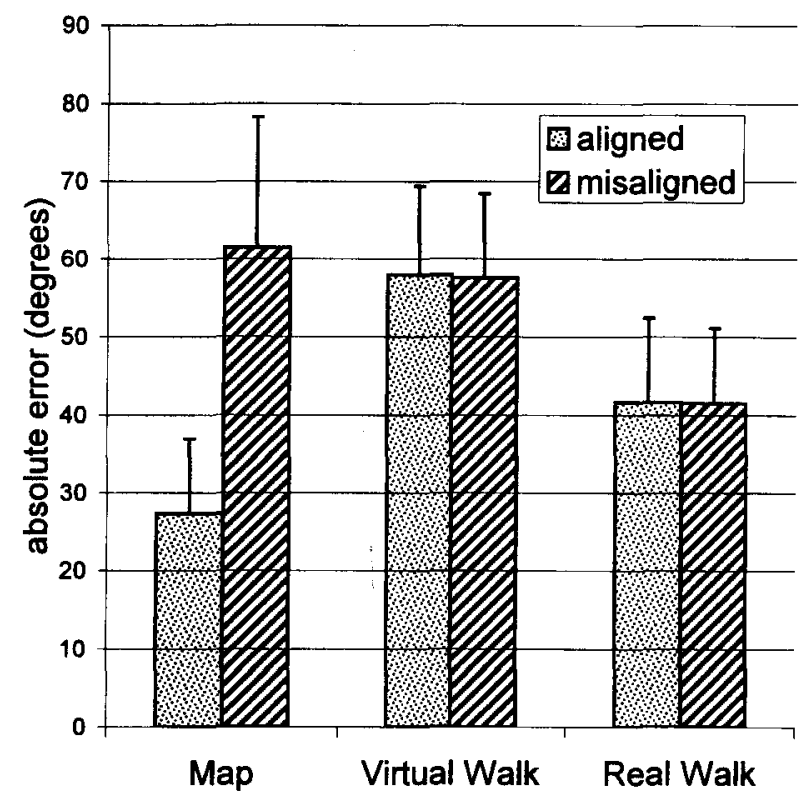

Figure 5. Absolute pointing error for aligned versus misaligned map orientation (error bars represent one standard error). 


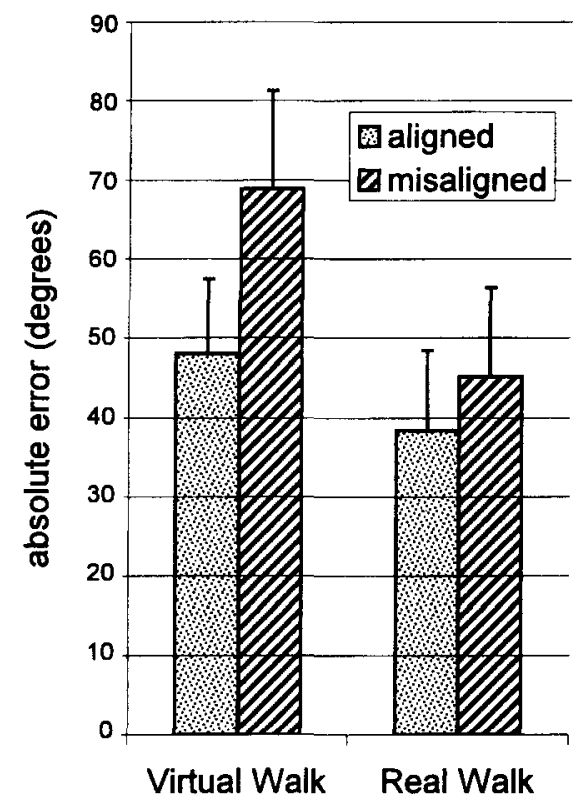

Figure 6. Absolute pointing error for aligned versus misaligned starting position orientation (error bars represent one standard error).

in making route distance estimates than in making straightline distance estimates.

Route distance. The correlation between objective and estimated route distance did not vary with learning condition $(p>.05)$. However, there was an interaction between learning condition and whether estimates were made between or within floors $[F(2,58)=4.03, p<.05]$. Post hoc comparisons on individual means were not significant, but the general trend indicated that map learners had higher within-floor route distance correlations than between-floor route distance correlations (.80 vs. .66), but this difference was not found with the other learning conditions.

Straight-line distance. As is shown in Figure 7, learning condition had a significant effect on straight-line distance estimates $[F(2,58)=11.45, p<.001]$. Post hoc comparisons indicated that the map learners had a higher correlation than did the VE and real-walk learners, and that the difference between VE and the real-walk learners was not significant. This is not surprising, given that straight-line distance requires a survey-level representation, which is depicted explicitly in a map but must be inferred from the route taken in the VE and real-walk conditions.

\section{Simple Virtual Environment Performance as a Predictor of Complex Environment Performance}

The participants gave directional judgments, straightline distance judgments, and route distance judgments for the simple VE task, as they did for the complex environment. However, since only six route and six straight-line estimates were made in the simple VE, there were not enough data to calculate a reliable correlation between objective and estimated distance. Therefore, directional estimates provided the primary measure of performance on the simple VE task, since they represent a mean rather than a correlation. The mean absolute error for these directional estimates was $30.6^{\circ}\left(S D=17.2^{\circ}\right)$.

The correlations between absolute pointing error in the simple VE task and in the complex environment task were computed for each of the three complex environment learning conditions. These correlations were $: 53(p<.05)$ for the map condition, $.64(p<.01)$ for the real-walk condition, and .60 $(p<.01)$ for the virtual-walk condition.

\section{Questionnaire Measures}

The participants reported having been on campus for an average of 2 years $(S D=1.61)$. They rated their familiarity with the complex environment (Ellison Hall) as $1.7(S D=1.09)$ and their familiarity with playing 3-D virtual reality games as $2.3(S D=1.89)$ on a scale of 1 to 7 , where $1=$ very unfamiliar and $7=$ very familiar.

\section{DISCUSSION}

In this study, we compared spatial representations of environments constructed from maps and from navigation in real environments and VEs. There were qualitative differences between the representations, resulting from the three forms of learning. To discuss these results, we first consider differences in spatial knowledge acquired from maps and from real-world navigation, and then we consider differences in spatial knowledge acquired from

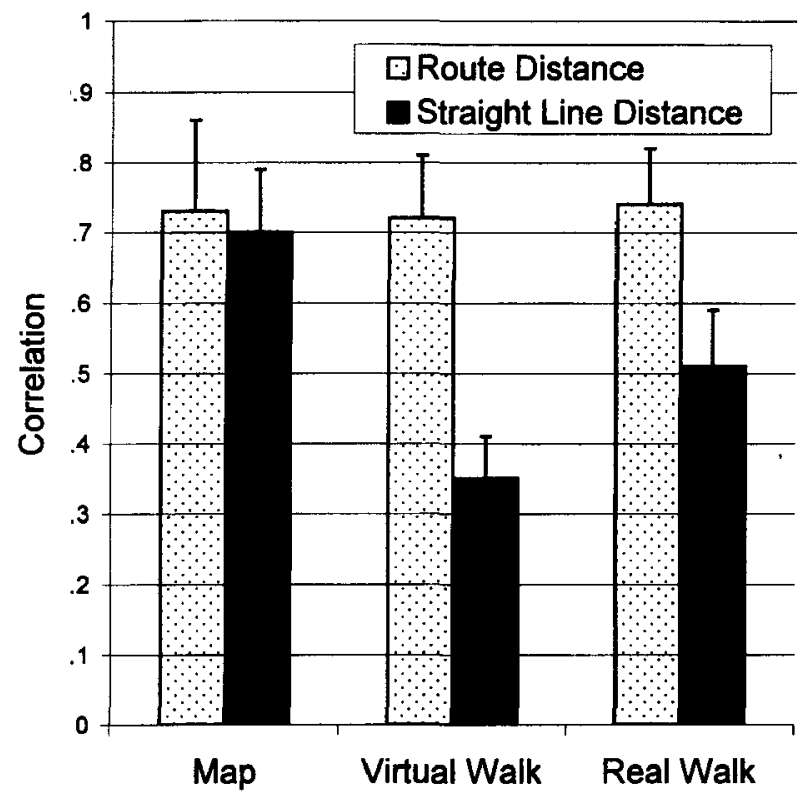

Figure 7. Correlation between objective distance and estimated distance (error bars represent one standard error). 
real environments and VEs. Finally, we consider how spatial learning from a VE predicts spatial learning from other experiences.

\section{Differences in Knowledge Acquired From Maps and Navigation}

One of the goals of this study was to compare the spatial representations learned from maps and from navigation. Averaging across all conditions of the experiment, no significant difference in absolute pointing error was found between the map and the navigation learning conditions. Furthermore, no differences in route distance correlations were found between map learners and realwalk learners. These results are inconsistent with Thorndyke and Hayes-Roth (1982), who found that navigators were superior to map learners in these respects. These discrepancies can be attributed to the large difference in exposure times between the two studies. The navigators in the Thorndyke and Hayes-Roth study had worked in the building 1-24 months, whereas the map learners studied the map for an hour or less. Our study suggests that, with equal amounts of exposure, levels of learning from a map and from navigation are more equivalent.

Consistent with previous studies, our results suggest that maps are powerful for acquiring quick and accurate survey knowledge, but knowledge that tends to be orientation specific. Total map exposure was only $6 \mathrm{~min}$, yet the participants exhibited remarkably accurate spatial knowledge when tested in an orientation that was aligned with the map. When tested in a misaligned orientation, their performance plummeted. Navigators had equivalent levels of performance in these two situations, confirming that the difference was a true alignment effect and not an experimental artifact (i.e., that the landmarks tested in the misaligned condition were more difficult to locate for some other reason). These results lend further support to the alignment effect literature (Evans \& Pezdek, 1980; Levine et al., 1984) and reveal an additional weakness in the Thorndyke and Hayes-Roth (1982) study. In their study, participants made orientation judgments from positions aligned or semi-aligned with the original map, and none with misalignment greater than $45^{\circ}$. It is likely that testing within the environment from misaligned orientations would have changed their findings substantially. Thorndyke and Hayes-Roth suggest that the orientation difficulty ascribed to map-derived knowledge is due to translating the overhead perspective of the map to the horizontal perspective of the environment. Orientation specificity from map-derived knowledge was never considered.

Although the orientation of the map affects the ability of map learners to make direction estimates, it does not affect straight-line distance estimates. Such estimates can be achieved if knowledge of the configuration is available, even without knowledge of one's heading with respect to that configuration. The straight-line distance results follow exactly the pattern of pointing errors for the aligned map condition, in that map learners showed higher correlations between real and estimated distance than did the other groups.

There were also differences in pointing accuracy, depending on whether people were tested from imagination or in situ. Map learners had a much greater difference in error between imagination and in situ responses (a difference of $26^{\circ}$ ) than did real-walk learners (a difference of $12^{\circ}$ ). These findings may be interpreted in two ways. First, testing map learners in the real environment might be interpreted as giving them a second learning trial (in which they learn from a real walk), confounding their map-derived knowledge with real-walk-derived knowledge. Second, during the learning phase, map participants were shown the route and the direction they would be facing before each landmark with an arrow. This directional arrow added a memory load for the map participants and may have led to confusion during testing.

The ability to integrate information on two different floors of a building into a single representation has not previously been studied in experiments comparing learning from maps and from navigation. Neither the map nor the real-walk learners showed a significant increase in error when pointing between floors, as compared with pointing within floors, demonstrating an ability to integrate two floors easily.

\section{Differences in Knowledge Acquired From Real and Virtual Environments}

This study provides important original data about the differences in spatial representations acquired from navigation in real environments and VEs. Consistent with previous studies (Philbin et al., 1998; Witmer et al., 1996), spatial learning was generally poorer for participants who learned from a VE. These results are at odds with Ruddle et al. (1997), who found similar levels of performance between their VE learners and Thorndyke and Hayes-Roth's (1982) navigators. However, in this study, $\mathrm{VE}$ and real-walk learners showed similar performance (less than $5^{\circ}$ difference in error) when pointing between landmarks on a single floor. Although the Thorndyke and Hayes-Roth environment was much larger than our environment, it was situated on a single floor. In a simple, single-floor environment, it seems that individuals might be able to acquire as much knowledge from learning in a VE as from learning in a real environment. It may be that VE participants had difficulty integrating the two floors or, more likely, that they were unable to correctly update their heading while traversing the eight $90^{\circ}$ turns in the stairwell. If their heading was not correctly updated, they may have not apprehended the overlapping floors, which is manifest in the real environment. In addition, Ruddle et al.'s VE learners received 10 learning trials (as opposed to a single trial in this study), and as in Thorndyke and Hayes-Roth, testing always occurred from an orientation aligned with the original starting orientation, which may have facilitated pointing. Realwalk learners in this study were led to the starting point of the walk, allowing them to see the outside shape of the 
building and, perhaps, giving them a further advantage in orienting.

A substantial alignment effect $\left(21^{\circ}\right.$ difference in error) was found for the VE navigators, indicating that they had learned the route with a preferred orientation corresponding to their original facing direction in the environment. This contrasts with Tlauka and Wilson's (1996) study, which found no alignment effects in VEs. However, Tlauka and Wilson only examined map alignment effects, which were meaningless in their virtual learning environment. In fact, in their study, the initial starting position for VE learners was contra-aligned with the map orientation; examination of their data suggests a trend for VE learners to show greater error when pointing from a position that was misaligned with this starting orientation.

Why would an alignment effect arise in the virtual but not in the real-walk conditions? A likely explanation is that both vestibular information and visual information are necessary to induce egocentric updating and that there is a lack of vestibular information in a desktop VE. Two recent studies varying the type of sensory information available to navigators lend support to this argument (Chance, Gaunet, Beall, \& Loomis, 1998; Klatzky, Loomis, Beall, Chance, \& Golledge, 1998). These studies show a significant decrease in orientation performance using visual information alone (without concomitant body rotation). In addition, Bakker, Werkhoven, and Passenier (1998) found that participants attempting to rotate $270^{\circ}$ in a VE using visual information alone undershot the rotation by $120^{\circ}$ (as compared with to a $25^{\circ}$ undershoot with vision and proprioceptive information combined). Studies that have examined imagined updating have shown similar results (e.g. Presson \& Montello, 1994; Rieser, 1989; Rieser, Guth, \& Hill, 1986). These studies have shown that imagined rotations are very difficult, but that imagined translations are as quick and accurate as actual translations. The difference between VE and real-walk performance was significantly smaller during in situ responses $\left(10^{\circ}\right)$ than during imagination responses $\left(22^{\circ}\right)$. Unlike imagination testing, during in situ testing, participants walked and turned in the environment, enabling them to update heading.

Route distance correlations did not differ between the VE and the real-walk conditions, indicating that such knowledge acquisition is as attainable in a $\mathrm{VE}$ as it is in a real environment. These results are reasonable, since relative route distance can be estimated simply by time passed. Since one receives no vestibular information during desktop virtual travel, these findings suggest that vestibular information is not critical for relative distance knowledge acquisition (at least under these circumstances). The differences between the straight-line distance measures for the VE and the real-walk conditions follow the same pattern as the absolute pointing error data. However, only the main effect of learning condition was significant.

In summary, acquiring configurational knowledge in a desktop VE is generally more difficult than in a real environment. Our results suggest that VE learners are par- ticularly susceptible to disorientation after rotation, as is indicated by the existence of a preferred environmental alignment and by their inability to point between floors after multiple turns. In terms of Presson and Hazelrigg's (1984) distinction between primary (direct) and secondary (through a symbolic medium) learning, VEs exhibit characteristics of both - representations are not as accurate as map-derived knowledge (such as that from secondary learning) and not as flexible as knowledge derived from direct experience (such as that from primary learning). Whether these representations are qualitatively different remains unclear. Although VE learners had difficulty adopting different orientations within the environment, it is possible that they were able to construct an accurate allocentric representation that was not orientation specific. A preferred orientation might be the orientation faced most often during VE navigation, which corresponds to the starting orientation in this study. Furthermore, it is unknown whether extensive training would reduce or eliminate that effect or whether the relative impoverishment of the VE also influenced our findings. These are empirical questions that should be considered in subsequent studies. In any case, these findings suggest that difficulty in updating heading after rotation may be an intrinsic aspect of learning in desktop VEs.

\section{Predicting Individual Differences \\ in Environmental Spatial Learning}

Although knowledge representations derived from VEs are not equivalent to those derived from real environments, a strong correlation exists in the ability to learn these two types of environments. This suggests the use of coincident cognitive mechanisms. Other attempts have been made to predict large-scale environmental ability from performance on various aptitude tests, such as the Vandenberg Mental Rotation Task and the Embedded Figures Test. However, these paper-and-pencil psychometric measures have very weak predictive power over large-scale spatial ability-usually with correlations between .05 and .30 that often fail to reach significance (see, e.g., Bryant, 1982; Cooper \& Mumaw, 1985). In fact, no other laboratory activity or battery of activities has shown a closer relationship to environmental learning ability. However, the higher correlation in our study probably reflects, to some extent, the similarity of the predictor and the criterion measures (i.e., pointing performance). Further research is needed to determine which aspects of acquisition are most likely to be shared processes. Since the relationship is based solely on pointing error-a strong measure of configurational knowledge-this suggests that the ability to update egocentric orientation is one of the shared mechanisms. As a practical point, VE learning performance may be a simple way of measuring environmental spatial ability in the laboratory setting.

\section{Conclusion}

The results of this study are threefold. First, although individuals were able to acquire a substantial amount of spatial knowledge from a VE, important differences in 
spatial representations were found after learning from real environments and from VEs. The existence of a VE alignment effect suggests that the optic flow specifying movement in a desktop VE is sufficient for translational but not for rotational updating. Second, a large alignment effect was found after learning the environment from a map, consistent with previous studies. It should be emphasized that map learning leads to superior performance only when aligned with the initial orientation of the map. The resulting representation is precise, yet inflexible. Finally, our results suggest that the cognitive processes necessary for maintaining orientation in a real environment are used, at least in part, when operating in a virtual one.

\section{REFERENCES}

Alfano, P. L., \& Michel, G. F. (1990). Restricting the field of view: Perceptual and performance effects. Perceptual \& Motor Skills, 70, 35-45.

Bakker, N. H., Werkhoven, P. J., \& Passenier, P. O. (1998). Aiding orientation performance in virtual environments with proprioceptive feedback. Proceedings of the IEEE 1998 Virtual Reality Annual International Symposium (pp. 28-33). Los Alamitos, CA: IEEE Computer Society Press.

BLISs, J. P., TidwELl, P. D., \& GuEst, M. A. (1997). The effectiveness of virtual reality for administering spatial navigation training to firefighters. Presence-Teleoperators \& Virtual Environments, 6, 73-86.

Bryant, K. J. (1982). Personality correlates of sense of direction and geographic orientation. Journal of Personality \& Social Psychology, 43, 1318-1324.

Chance, S. S., Gaunet, F., Beall, A. C., \& Loomis, J. M. (1998). Locomotion mode affects the updating of objects encountered during travel: The contribution of vestibular and proprioceptive inputs to path integration. Presence-Teleoperators \& Virtual Environments, 7, 168-178.

CoOPER, L. A., \& Mumaw, R. J. (1985). Spatial aptitude. In R. F. Dillon (Ed.), Individual differences in cognition (Vol. 2, pp. 67-94). Orlando, FL: Academic Press.

DARKEN, R. P., \& SIBERT, J. L. (1996). Navigating large virtual spaces. International Journal of Human-Computer Interaction, 8, 49-71.

Evans, G. W., \& PEZdeK, K. (1980). Cognitive mapping: Knowledge of real-world distance and location information. Journal of Experimental Psychology: Human Learning \& Memory, 6, 13-24.

Goldin, S. E., \& THORNDYKE, P. W. (1981). An analysis of cognitive mapping skill (N-1664-ARMY). Santa Monica, CA: The Rand Corporation.

GoldiN, S. E., \& THORNDYKe, P. W. (1982). Simulating navigation for spatial knowledge acquisition. Human Factors, 24, 457-471.

HENRY, D., \& FuRNEss, T. (1993). Spatial perception in virtual environments: Evaluating an architectural application. Proceedings of the IEEE Virtual Reality Annual Symposium (pp. 33-40). Piscataway, NJ: IEEE Service Center.

Klatzky, R. L., Loomis, J. M., Beall, A. C., Chance, S. S., \& GolLEDGE, R. G. (1998). Spatial updating of self-position and orientation during real, imagined, and virtual locomotion. Psychological Science, 9, 293-298.

LeVine, M., MarChon, I., \& HANLEY, G. L. (1984). The placement and misplacement of you-are-here maps. Environment \& Behavior, 16, 139-157.

LLOYD, R., \& CAMmaCK, R. (1996). Constructing cognitive maps with orientation biases. In J. Portugali (Ed.), The construction of cognitive maps (pp. 187-213). Dordrecht: Kluwer.

LORENZ, C. A., \& NEISSER, U. (1986). Ecological and psychometric dimensions of spatial ability (Rep. No. 10). Atlanta: Emory University, Emory Cognition Project.

MACEACHREN, A. M. (1992). Learning spatial information from maps:
Can orientation specificity be overcome? Professional Geographer, 44, $431-443$

Montello, D. R. (1991). The measurement of cognitive distance: Methods and construct validity. Journal of Environmental Psychology, 11, 101-122.

PaliJ, M., Levine, M., \& Kahan, T. (1984). The orientation of cognitive maps. Bulletin of the Psychonomic Society, 22, 105-108.

PEARSON, J. L., \& IAlONGo, N. S. (1986). The relationship between spatial ability and environmental knowledge. Journal of Environmental Psychology, 6, 299-304.

Philbin, D. A., Ribarsky, W., Walker, N., \& Hubbard, C. E. (1998). Training in virtual environments: Analysis of task appropriateness. Proceedings of the IEEE 1998 Virtual Reality Annual International Symposium (p. 210). Los Alamitos, CA: IEEE Computer Society Press.

Presson, C. C., Delange, N., \& Hazelrigg, M. D. (1987). Orientationspecificity in kinesthetic spatial learning: The role of multiple orientations. Memory \& Cognition, 15, 225-229.

Presson, C. C., Delange, N., \& Hazelrigg, M. D. (1989). Orientationspecificity in spatial memory: What makes a path different from a map of the path? Journal of Experimental Psychology: Learning, Memory, \& Cognition, 15, 887-897.

Presson, C. C., \& HazelRigG, M. D. (1984). Building spatial representations through primary and secondary learning. Journal of Experimental Psychology: Learning, Memory, \& Cognition, 10, 716-722.

PREsSON, C. C., \& MONTELLO, D. R. (1994). Updating after rotational and translational body movements: Coordinate structure of perspective space. Perception, 23, 1447-1455.

Regian, J. W., ShebilsKe, W. L., \& Monk, J. M. (1992). Virtual reality: An instructional medium for visual-spatial tasks. Journal of Communication, 42, 136-149.

RIESER, J. J. (1989). Access to knowledge of spatial structure at novel points of observation. Journal of Experimental Psychology: Learning, Memory, \& Cognition, 15, 1157-1165.

RIESER, J. J., GUTH, D. A., \& Hill, E. W. (1986). Sensitivity to perspective structure while walking without vision. Perception, 15, 173-188.

Ruddle, R. A., PAYne, S. J., \& Jones, D. M. (1997). Navigating buildings in "desk-top" virtual environments: Experimental investigations using extended navigational experience. Journal of Experimental Psychology: Applied, 3, 143-159.

SchuTZ, R. W., \& Roy, E. A. (1973). Absolute error: The devil in disguise. Journal of Motor Behavior, 5, 141-153.

Shelton, A. L., \& MCNAMARA, T. P. (1997). Multiple views of spatial memory. Psychonomic Bulletin \& Review, 4, 102-106.

SHOLL, M. J. (1996). From visual information to cognitive maps. In J. Portugali (Ed.), The construction of cognitive maps (pp. 157-186). Dordrecht: Kluwer

ThoRndyKe, P. W., \& HAyes-Roth, B. (1982). Differences in spatial knowledge acquired from maps and navigation. Cognitive Psychology, 14, 560-589.

TLAUKA, M., \& WILSON, P. N. (1996). Orientation-free representations from navigation through a computer-simulated environment. Environment \& Behavior, 28, 647-664.

WARREN, W. H., \& HANNON, D. J. (1988). Direction of self-motion is perceived from optical flow. Nature, 336, 162-163.

WICKENS, C. D., \& BAKER, P. (1995). Cognitive issues in virtual reality. In T. A. F. Woodrow Barfield III (Ed.), Virtual environments and advanced interface design (pp. 514-541). New York: Oxford University Press.

Witmer, B. G., Bailey, J. H., KNERr, B. W., \& Parsons, K. C. (1996). Virtual spaces and real world places: Transfer of route knowledge. $I n$ ternational Journal of Human-Computer Studies, 45, 413-428.

WitMER, B. G., \& KLINE, P. B. (1998). Judging perceived and traversed distance in virtual environments. Presence-Teleoperators \& Virtual Environments, 7, 144-167.

(Manuscript received November 25, 1997; revision accepted for publication August 7,1998 .) 